\title{
XII. Ueber die Isomorphie des Natriumcarbonats mit dem Natriumsulfit.
}

Von

Hermann Traube in Berlin.

Auf die unverkennbare Analogie der Alkalicarbonate mit den Alkalisulfiten, welche besonders bei den Natriumsalzen im Krystallwassergebalt hervortritt, ist schon mehrfach hingewiesen worden*). Man kennt beim Natriumcarbonat Verbindungen mit 1 Mol., 3 Mol., 5 Mol., 6 Mol., 7 Mol., $10 \mathrm{Mol}$. und $15 \mathrm{Mol}$. Krystallwasser, beim Natriumsulfit ausser dem wasserfreien Salz Verbindungen mil $7^{* *}$ ) und 10**) Mol. Krystallwasser. $\mathrm{Na}_{2} \mathrm{CO}_{3}$ $+7 \mathrm{H}_{2} \mathrm{O}$ krystallisirt rhombisch $\mathrm{t}_{\text {) }}, \mathrm{Na}_{2} \mathrm{SO}_{3}+7 \mathrm{H}_{2} \mathrm{O}$ monosymmetrisch $\left.+\mathrm{t}\right)$, $\mathrm{Na}_{2} \mathrm{CO}_{3}+10 \mathrm{H}_{2} \mathrm{O}$ monosymmetrisch $+\dagger+$ ), $\mathrm{Na}_{2} \mathrm{SO}_{3}+10 \mathrm{H}_{2} \mathrm{O}$ ist krystallographisch noch nicht untersucht worden. Die Verschiedenheit der Krystallform bei den mit $7 \mathrm{Mol} . \mathrm{H}_{2} \mathrm{O}$ krystallisirenden Salzen ist wohl der Grund, dass man es bisher auffälliger Weise unterlassen hat, zu prufen, ob diese Substanzen im Stande sind Mischkrystalle zu bilden. Darauf hingehende Versuche liessen sofort erkennen, dass dies sowohl bei den mit 10, als auch bei den mit $7 \mathrm{Mol}$. $\mathrm{H}_{2} \mathrm{O}$ krystallisirenden Natriumsalzen der Kohlensäure und schwefligen Säure der Fall ist. Auf die Bestimmung der Löslichkeit dieser Mischkrystalle, wie sie H. W. Back hu is Roozeboom *t) beim chlorsauren Kali $\mathrm{KClO}_{3}$ und chlorsauren Thallium $\mathrm{TlClO}_{3}$ ausfuhrte, wurde hier

*) Vergl. unter Anderen besonders Muspratt, Ann. d. Chem. u. Pharm. 50, 259 und Röhrig, Journ. f. prakt. Chem. 1887 (2), 37, 217.

**) Röhrig, Journ. f. prakt. Chem. 1887 (2), 37, 223. Rammels berg, Handbuch d. kryst.-phys. Chem. 1, 471 hatte nur 6 Mol. $\mathrm{H}_{2} \mathrm{O}$ angenommen.

***) Muspratt, Ann. d. Chern. u. Pharm. 50, 229.

t) R a mmels berg, Handb. der kryst.-phys. Chemie 1, 348.

t+) Rammelsberg, l. c. 471. Marignac, Ann. d. mines (5), 12, 30.

ttt) Mohs, Pogg. Ann. 5, 369.

${ }^{*}+$ ) H. W. Bakbu is Ro oze boom, Zeitschr. f. physikal. Chem. 1891, 8, э31. 
zunächst noch nicht eingegangen, sondern einstweilen nur die Zusammensetzung der Mischkrystalle selbst festgestellt. Eine vollständige Ermittelung der krystallographischen Gonstanten liess sich, da die Mischkrystalle ungemein rasch an der Luft verwittern, nicht in allen Fällen ausführen, bisweilen konnten nur zwei Winkel gemessen und einmal musste von einer goniometrischen Untersuchung uberhaupt abgesehen werden.

Die Analysen wurden in der Weise ausgeführt, dass in der in Wasser gelösten Substanz die schweflige Säure durch Bromwasser oxydirt und die Schwefelsäure, nachdem die Lösung unter Zusatz von concentrirter Chlorwasserstoffsäure bis zur Entfärbung gekocht war, als Baryumsulfat gewogen wurde; im Filtrat wurde nach Ausfällung des uberschüssigen Chlorbaryums das Natrium als Sulfat bestimmt. Eine directe Bestimmung der Kohlensäure erfolgte nur in einem Falle; eine besondere Menge wasserfreien Salzes wurde mit Kaliumbichromat gegluht und die entweichende Kohlensäure von Kalilauge aufgenommen.

\section{Mischkrystalle mit 10 Mol. Krystallwasser.}

1) Eine Lösung, welche auf $1 \mathrm{Mol}$. (wasserfreies) $\mathrm{Na}_{2} \mathrm{CO}_{3} \quad 0,206 \mathrm{Mol}$. $\mathrm{Na}_{2} \mathrm{SO}_{3}$ enthielt, gab Krystalle, welche der Zusammensetzung $1 \mathrm{Mol} . \mathrm{Na}_{2} \mathrm{CO}_{3}$ $+10 \mathrm{H}_{2} \mathrm{O}+0,133 \mathrm{Na}_{2} \mathrm{SO}_{3}+10 \mathrm{H}_{2} \mathrm{O}$ entsprachen. Spec. Gew. $=1,501$.

Angewandt 1,083 g Substanz; gefunden 0,103 $\mathrm{BaSO}_{4}, 0,537 \mathrm{Na}_{2} \mathrm{SO}_{4}$, 0,674. $\mathrm{H}_{2} \mathrm{O}$.

\begin{tabular}{lrc} 
& Gelunden: & Berechnet: \\
$\mathrm{SO}_{2}$ & 2,61 & 2,60 \\
$\mathrm{CO}_{2}$ & 14,61 & 13,43 \\
$\mathrm{Na}_{2} \mathrm{O}$ & 21,53 & 21,75 \\
$\mathrm{H}_{2} \mathrm{O}$ & 62,25 & 62,22 \\
\hline & 100,00 & 100,00
\end{tabular}

Die Krystalle hatten die Form des Salzes $\mathrm{Na}_{2} \mathrm{CO}_{3}+10 \mathrm{H}_{2} \mathrm{O}$ und zeigten die Formen $\{010\},\{111\},\{110\}$, sie waren nach (010) dicktafelförmig ausgebildet; an ihnen wurde gemessen:

$$
\begin{array}{lc} 
& \left.\mathrm{Mohs}^{*}\right) \text { gemessen an } \mathrm{Na}_{2} \mathrm{CO}_{3}+10 \mathrm{H}_{2} \mathrm{O} \\
(\mathrm{T} 11):\left(T_{T} \mathrm{~T}\right)=76^{0} \mathrm{52}^{\prime} & 76^{0} 28^{\prime} \\
(110):(1 T 0)=80 \text { 20 } & 7940
\end{array}
$$

2) Eine Lösung, welche auf $1 \mathrm{Mol} . \mathrm{Na}_{2} \mathrm{CO}_{3}$ 0,437 $\mathrm{Mol} . \mathrm{Na}_{2} \mathrm{SO}_{3}$ enthielt, gab Krystalle, welche der Zusammensetzung: $1 \mathrm{Mol} . \mathrm{Na}_{2} \mathrm{CO}_{3}+10 \mathrm{H}_{2} \mathrm{O}$ $+0,182 \mathrm{Mol}$. $\mathrm{Na}_{2} \mathrm{SO}_{3}+10 \mathrm{H}_{2} \mathrm{O}$ entsprachen. Spec. Gew. $=1,5025$.

Angewandte Substanz 2,705, gefunden 0,315 $\mathrm{BaSO}_{4}, 1,330 \mathrm{Na}_{2} \mathrm{SO}_{4}$, $1,686 \mathrm{H}_{2} \mathrm{O}$.

*) Mohs, Pogg. Ann. 5, 369. 


\begin{tabular}{lcc} 
& Gefunden : & Berechnet: \\
$\mathrm{SO}_{2}$ & 3,20 & 3,40 \\
$\mathrm{CO}_{2}$ & 13,01 & 12,87 \\
$\mathrm{Na}_{2} \mathrm{O}$ & 21,46 & 21,48 \\
$\mathrm{H}_{2} \mathrm{O}$ & 62,33 & 62,25 \\
\hline & 100,00 & 100,00
\end{tabular}

Die Krystalle besassen dieselbe Ausbildung und zeigten dieselben Formen wie die unter 1 beschriebenen; an ihnen wurde gemessen:

$$
\begin{array}{cc} 
& \text { Mohs gemessen an } \mathrm{Na}_{2} \mathrm{CO}_{3}+10 \mathrm{H}_{2} \mathrm{O} \\
76^{\circ} 50^{\prime} & 76^{\circ} 28^{\prime} \\
8016 & 7940
\end{array}
$$

Aus einer Lösung, welche auf $1 \mathrm{Mol} . \mathrm{Na}_{2} \mathrm{CO}_{3} \quad 0,875 \mathrm{Mol} . \mathrm{Na}_{2} \mathrm{SO}_{3}$ enthielt, konnten bei $20^{\circ} \mathrm{C}$. Krystalle mit ${ }^{0} \mathrm{H}_{2} \mathrm{O}$ nicht erhalten werden.

Mischkrystalle mit 7 Mol. Krystallwasser.

3) Eine Lösung, welche auf $1 \mathrm{Mol} . \mathrm{Na}_{2} \mathrm{CO}_{3}$ (wasserfrei) 0,165 Mol. $\mathrm{Na}_{2} \mathrm{SO}_{3}$ enthielt, gab Krystalle, welche der Zusammensetzung $1 \mathrm{Mol} . \mathrm{Na}_{2} \mathrm{CO}_{3}$ $+{ }_{7} \mathrm{H}_{2} \mathrm{O}+0,067 \mathrm{Mol} . \mathrm{Na}_{2} \mathrm{SO}_{3}+7 \mathrm{H}_{2} \mathrm{O}$ entsprechen. Spec. Gew. 1,321.

Angewandte Substanz 2,882 $\mathrm{g}$, gefunden 0,183 $\mathrm{BaSO}_{4}, 1,755 \mathrm{Na}_{2} \mathrm{SO}_{4}$, $1,557 \mathrm{H}_{2} \mathrm{O}$.

\begin{tabular}{lcc} 
& Gefunden: & Berechnet: \\
$\mathrm{SO}_{2}$ & 1,75 & 1,72 \\
$\mathrm{CO}_{2}$ & 17,60 & 17,68 \\
$\mathrm{Na}_{2} \mathrm{O}$ & 26,53 & 26,58 \\
$\mathrm{H}_{2} \mathrm{O}$ & 54,03 & 54,02 \\
\hline & 100,00 & 100,00
\end{tabular}

Die rhombischen Krystalle zeigen die Form des Salzes $\mathrm{Na}_{2} \mathrm{CO}_{3}+7 \mathrm{H}_{2} \mathrm{O}$ und lassen die Formen $\{010\},\{100\},\{111\},\{021\},\{230\}$ erkennen, sie sind nach (010) dünntafelförmig, es ist:

$$
\begin{aligned}
& a: b: c=0,73782: 1: 0,35565 \\
& =0,751: 1: 0,360 \quad \mathrm{Na}_{2} \mathrm{CO}_{3}+7 \mathrm{H}_{2} \mathrm{O} \text { Rammelsberg*). } \\
& \text { Gemessen : } \\
& (021):(0 \overline{2} 1) \quad 71054 \\
& \text { Berechnet: Rammelsberg ber.: } \\
& \text { (111):(T11) } 36 \quad 12 \\
& \text { - } \quad 71034^{\prime} \\
& (010):(021) \quad 54 \quad 6 \\
& \begin{array}{rrr}
54^{0} 3^{\prime} & 36 & 0 \\
& 54 & 13
\end{array}
\end{aligned}
$$

4) Eine Lösung, welche auf $1 \mathrm{Mol} . \mathrm{Na}_{2} \mathrm{CO}_{3}$ 0,206 Mol. $\mathrm{Na}_{2} \mathrm{SO}_{3}$ enthielt, gab Krystalle, welche der Zusammensetzung $1 \mathrm{Mol} . \mathrm{Na}_{2} \mathrm{CO}_{3}+7 \mathrm{H}_{2} \mathrm{O}+0,454$ $\mathrm{Na}_{2} \mathrm{SO}_{3}+7 \mathrm{H}_{2} \mathrm{O}$ entsprachen. Spec. Gew. 1,531.

*) Rammelsberg, Handbuch der kryst.-phys. Chemie 1, 548. 
Angewandte Substanz 3,674 g, gefunden $1,136 \mathrm{BaSO}_{4}, 2,195 \mathrm{Na}_{2} \mathrm{SO}_{4}$, $1,947 \mathrm{H}_{2} \mathrm{O}$.

\begin{tabular}{lcc} 
& Gefunden: & Berechnet: \\
$\mathrm{SO}_{2}$ & $\mathbf{8 , 4 9}$ & $\mathbf{8 , 4 0}$ \\
$\mathrm{CO}_{2}$ & 12,42 & 12,70 \\
$\mathrm{Na}_{2} \mathrm{O}$ & 26,09 & 26,02 \\
$\mathrm{H}_{2} \mathrm{O}$ & 52,99 & 52,88 \\
\hline & 100,00 & 100,00
\end{tabular}

Die Krystalle besassen dieselbe Ausbildung und zeigten dieselben Formen wie die unter 3) beschriebenen. Die Messungen an den ungemein rasch trube werdenden Krystallen waren nicht sehr genau.

$$
\begin{aligned}
& a: b: c=0,78057: 1: 0,36370 \\
& =0,751: 1: 0,360 \quad \mathrm{Na}_{2} \mathrm{CO}_{3}+7 \mathrm{H}_{2} \mathrm{O} \text { Rammelsberg. } \\
& \text { Gemessen: Rammelsberg: } \\
& \begin{array}{lll}
(010):(021) & 53058^{\prime} & 54013^{\prime} \\
(010):(230) & 3030 & 3136
\end{array}
\end{aligned}
$$

5) Eine Lösung, welche auf $1 \mathrm{Mol} . \mathrm{Na}_{2} \mathrm{CO}_{3}$ 0,240 Mol. $\mathrm{Na}_{2} \mathrm{SO}_{3}$ enthielt, gab Krystalle, welche der Zusammensetzung 1 Mol. $\mathrm{Na}_{2} \mathrm{CO}_{3}+7 \mathrm{H}_{2} \mathrm{O}$ $+0,5 \mathrm{Mol} . \mathrm{Na}_{2} \mathrm{SO}_{3}+7 \mathrm{H}_{2} \mathrm{O}$ entsprechen. Spec. Gew. 1,550 .

Angewandte Substanz 1,357 g, gefunden 0,458 $\mathrm{BaSO}_{4}, 0,806 \mathrm{Na}_{2} \mathrm{SO}_{4}$, $0,716 \mathrm{H}_{2} \mathrm{O}$.

$1,908 \mathrm{~g}$ enthielten $0,2285 \mathrm{CO}_{2}$.

\begin{tabular}{lcc} 
& Gefunden: & Berechnet: \\
$\mathrm{SO}_{2}$ & 8,94 & 9,28 \\
$\mathrm{CO}_{2}$ & 14,98 & 12,07 \\
$\mathrm{Na}_{2} \mathrm{O}$ & 25,98 & 25,92 \\
$\mathrm{H}_{2} \mathrm{O}$ & 52,79 & 52,73 \\
\hline & 99,69 & 100,00
\end{tabular}

Die monosymmetrischen Krystalle hatlen die Form des Salzes $\mathrm{Na}_{2} \mathrm{SO}_{3}$ $+7 \mathrm{H}_{2} \mathrm{O}$, sie zeigten die Formen $\{001\},\{255\},\{230\},\{100\}$, waren in der Richtung der Axe stark ausgedehnt, $\{100\}$ besass stets nur geringe Ausdehnung. Die grossen Krystalle verwitterten so rasch an der Luft, dass nur mit dem Anlegegoniometer einige approximalive Messungen vorgenommen werden konnten. Es wurde gemessen:

$\begin{array}{ccc} & \text { Rammelsberg*) } \\ (255):(001) & 115^{0} & 114^{0} \\ (230):(2 \overline{3} 0) & 66 & 65\end{array}$

6) Eine Lösung, welche auf $1 \mathrm{Mol} . \mathrm{Na}_{2} \mathrm{CO}_{3} 1,033 \mathrm{Mol} . \mathrm{Na}_{2} \mathrm{SO}_{3}$ enthielt,

*) Ha m m e l s berg, Handbuch d. krystallogr.-phys. Chemie 1, 471. 
gab Krystalle, welche der Zusammensetzung $1 \mathrm{Mol} . \mathrm{Na}_{2} \mathrm{CO}_{3}+7 \mathrm{aq}+2 \mathrm{Mol}$. $\mathrm{Na}_{2} \mathrm{SO}_{3}+7 \mathrm{aq}$ entsprechen. Spec. Gew. 4,556 .

Angewandte Substanz 1,658 g. Gefunden 0,996 $\mathrm{BaSO}_{4}, 0,963 \mathrm{Na}_{2} \mathrm{SO}_{4}$, $0,855 \mathrm{H}_{2} \mathrm{O}$.

\begin{tabular}{lrc} 
& Gefunden: & Berechnet: \\
$\mathrm{SO}_{2}$ & 16,50 & 17,38 \\
$\mathrm{CO}_{2}$ & 6,58 & 5,99 \\
$\mathrm{Na}_{2} \mathrm{O}$ & 25,36 & 25,27 \\
$\mathrm{H}_{2} \mathrm{O}$ & 59,56 & 51,36 \\
\hline & 100,00 & 100,00
\end{tabular}

Die Krystalle zeigten die Form des Salzes $\mathrm{Na}_{2} \mathrm{SO}_{3}+7 \mathrm{H}_{2} \mathrm{O}$ und liessen die Flächen $\{001\},\{100\},\{255\},\{230\},\{101\}$ erkennen, sie waren nach (001) dicktafelförmig und nach den Axen $a$ und $b$ ungefäbr gleich ausgedehnt. Die Messungen ergaben:

$$
\begin{aligned}
& a: b: c=1,08953: 1: 0,75275 ; \quad \beta=92^{0} 88^{\prime} \\
& =1,048: 1: 0,765 ; \quad \beta=9336 \mathrm{Na}_{2} \mathrm{SO}_{3}+7 \mathrm{H}_{2} \mathrm{O} \\
& \text { Rammelsberg. } \\
& (110):(\overline{1} 10) \\
& \text { Gemessen : } \\
& (001):(101) \\
& 64010^{\prime} \\
& \text { Rammielsberg } \mathrm{Na}_{2} \mathrm{SO}_{3}+7 \mathrm{H}_{2} \mathrm{O} \text {. } \\
& (001):(100) \\
& 35 \quad 32 \\
& 65^{\circ} \quad 0^{\prime} \\
& 34 \quad 52 \\
& 87 \quad 12 \\
& 86 \quad 24
\end{aligned}
$$

7) Eine Lösung, welche auf $1 \mathrm{Mol} . \mathrm{Na}_{2} \mathrm{CO}_{3} 2,413 \mathrm{Mol} . \mathrm{Na}_{2} \mathrm{SO}_{3}$ enthielt, gab Krystalle, welche der Zusammensetzung $1 \mathrm{Mol} . \mathrm{Na}_{2} \mathrm{CO}_{3}+7 \mathrm{H}_{2} \mathrm{O}+4 \mathrm{Mol}$. $\mathrm{Na}_{2} \mathrm{SO}_{3}+7 \mathrm{H}_{2} \mathrm{O}$ entsprachen. Spec. Gew. 1,558.

Angewandte Substanz 2,921, gefunden 2,167 $\mathrm{BaSO}_{4}, 1,674 \mathrm{Na}_{2} \mathrm{SO}_{4}$, $1,485 \mathrm{H}_{2} \mathrm{O}$.

\begin{tabular}{lcr} 
& Gefunden: & Berechnet: \\
$\mathrm{SO}_{2}$ & 20,38 & 20,64 \\
$\mathrm{CO}_{2}$ & 3,66 & 3,56 \\
$\mathrm{Na}_{2} \mathrm{O}$ & 25,03 & 25,00 \\
$\mathrm{H}_{2} \mathrm{O}$ & 50,83 & 50,80 \\
\hline & 100,00 & 100,00
\end{tabular}

Die Krystalle zeigen dieselbe Ausbildung und dieselben Flächen wie Nr. 6. Die Messungen ergaben:

$$
\begin{aligned}
& a: b: c=1,08560: 1: 0,76230 ; \beta=92050^{\prime} \\
& =1,048: 1: 0,765 ; \quad \beta=9336 \mathrm{Na}_{2} \mathrm{SO}_{3}+\mathrm{H}_{2} \mathrm{O} \\
& \text { Rammelsberg. } \\
& (110):(\bar{T} 10) \\
& (001):(101) \\
& 64^{\circ} 24^{\prime} \\
& (001):(100) \\
& 36 \quad 0 \\
& \text { Rammelsberg } \mathrm{Na}_{2} \mathrm{SO}_{3}+7 \mathrm{H}_{2} \mathrm{O} \text {. } \\
& 65^{\circ} 0^{\prime} \\
& 34 \quad 52 \\
& 87 \quad 10 \\
& 86 \quad 24
\end{aligned}
$$


Aus den vorstehenden Untersuchungen geht ausser der Isomorphie der Salze $\mathrm{Na}_{2} \mathrm{CO}_{3}+10 \mathrm{H}_{2} \mathrm{O}$ mit $\mathrm{Na}_{2} \mathrm{SO}_{3}+10 \mathrm{H}_{2} \mathrm{O}$ die Isodimorphie von $\mathrm{Na}_{2} \mathrm{CO}_{3}+7 \mathrm{H}_{2} \mathrm{O}$ und $\mathrm{Na}_{2} \mathrm{SO}_{3}+7 \mathrm{H}_{2} \mathrm{O}$ hervor, denn die beiden zuletzt genannten Salze bilden sowohl rhombische, als auch monosymmetrische Mischkrystalle. Im freien Zustande ist vom Carbonat nur die rhombische, vom Sulfit nur die monosymmetrische Form bekannt.

Enthält eine Lösung auf $1 \mathrm{Mol} . \mathrm{Na}_{2} \mathrm{CO}_{3}$ mehr als 0,206 $\mathrm{Mol} . \mathrm{Na}_{2} \mathrm{SO}_{3}$, so gelangen nur monosymmetrische Krystalle zur Ausbildung. Ob die Verbindung $\mathrm{Na}_{2} \mathrm{SO}_{3}+10 \mathrm{H}_{2} \mathrm{O}$ bei niedriger Temperatur für sich allein in denselben Formen krystallisirt wie $\mathrm{Na}_{2} \mathrm{CO}_{3}+10 \mathrm{H}_{2} \mathrm{O}$, müssen spätere Untersuchungen zeigen. Weiterhin ergiebt sich, dass die Mischungsfähigkeit der Salze mit $10 \mathrm{Mol}$. Krystallwasser nicht dieselbe ist, wie die der mit $7 \mathrm{Mol}$. Aus einer Lösung, welche auf $1 \mathrm{Mol} . \mathrm{Na}_{2} \mathrm{CO}_{3}$ 0,206 Mol. $\mathrm{Na}_{2} \mathrm{SO}_{3}$ enthielt, schieden sich einmal Krystalle der Zusammensetzung $1 \mathrm{Mol} . \mathrm{Na}_{2} \mathrm{CO}_{3}+$ $10 \mathrm{H}_{2} \mathrm{O}+0,133 \mathrm{Mol} . \mathrm{Na}_{2} \mathrm{SO}_{3}+10 \mathrm{H}_{2} \mathrm{O}$, das andere Mal Krystalle der Zusammensetzung $1 \mathrm{Mol}$. $\mathrm{Na}_{2} \mathrm{CO}_{3}+7 \mathrm{H}_{2} \mathrm{O}+0,454 \mathrm{Mol} . \mathrm{Na}_{2} \mathrm{SO}_{3}+7 \mathrm{H}_{2} \mathrm{O}$ ab.

Die Isomorphie des Natriumcarbonats mit dem Natriumsulfit ist fúr die Constitution des letzteren nicht ohne Wichtigkeit. Man nimmt bekanntlich fur die schweflige Säure theils eine symmetrische Structurformel an, wobei dem Schwefel 4 Werthigkeiten zugeschrieben werden, theils eine asymmetrische, in der der Schwefel sechswerthig erscheint, an :

$$
O=\stackrel{\mathrm{IV}}{S}<\underset{O H}{O H}, \quad O=\underset{\mathrm{O}}{\mathrm{SI}}<\underset{O H}{H} .
$$

Nach der Isomorphie mit Natriumcarbonat scheint für dás Natriumsulfit wohl nur die Annahme einer symmetrischen Structurformel zulässig zu sein.

Eine den Alkalicarbonaten und Sulfiten analoge Zusammensetzung besitzen auch die Alkali-Stannate und Metasilicate, insbesondere $\mathrm{Na}_{2} \mathrm{SnO}_{3}$ und $\mathrm{Na}_{2} \mathrm{SiO}_{3}$. Ersteres krystallisirt gewöhnlich mit 3, unter gewissen Umständen aber auch mit $10 \mathrm{Mol}$. $\mathrm{H}_{2} \mathrm{O}^{*}$ ), letzteres mit 5, 6, 8 und $9 \mathrm{Mol} . \mathrm{H}_{2} \mathrm{O}$. Versuche, Mischkrystalle der hier zuletzt genannten Salze mit den entsprechenden Carbonaten und Sulfiten zu erhalten, haben kein sicheres Ergebniss geliefert. Bei Anwendung des stark verunreinigten, nicht krystallisirten, käuflichen Natriumstannats (Präparirsalz) bildeten sich aus Lösungen, welche gleichzeitig Natriumcarbonat enthielten, Krystalle von $\mathrm{Na}_{2} \mathrm{CO}_{3}$ mit 10 und auch mit $7 \mathrm{Mol}$. $\mathrm{H}_{2} \mathrm{O}$, die höchstens $2 \%$ des entsprechenden Stannats enthielten. Um eine etwa bestehende Isomorphie nachzuweisen, müssen Versuche mit reinem, schwer krystallisirt zu erhaltenden Natriumstannat angestellt werden.

*) Scheurer-Kestner, Bull. soc. chim. (2), 8, 389. 
Das Natriumhypophosphat bat zwar die empirische Zusammensetzung $\mathrm{Na}_{2} \mathrm{PO}_{3}+5 \mathrm{H}_{2} \mathrm{O}$, es bildet aber, wie Versuche zeigten, nicht Mischkrystalle mit Natriumcarbonat oder Natriumsulfit; es ist dies auch erklärlich, da man bekanntlich die Unterphosphorsäure als eine vierbasische Säure ansprechen und dem Natriumsalz daher die Formel $\mathrm{Na}_{4} \mathrm{P}_{2} \mathrm{O}_{6}+10 \mathrm{H}_{2} \mathrm{O}$ zuertheilen muss. Setzt man zu einer gesättigten Lösung von Natriumhypophosphat Natriumcarbonat zu, so scheidet sich ersteres augenblicklich vollkommen rein aus.

Demnächst sollen auch noch andere Carbonale und Sulfite auf Isomorphie untersucht werden.

Berlin, 21. Juni 4893. Zweites chemisches Institut der Universität. 